\title{
Tratamiento de la piedra, revestimientos y acabados
}

Fátima Bermúdez-Coronel García de Vinuesa

Restauradora

\section{Estado de conservación}

Toda intervención debe ir encaminada a devolver su funcionalidad a la obra, sin modificar ni alterar su naturaleza, apoyándose en una metodología de estudios específicos que ayuden a conocer su estado de conservación y el tratamiento adecuado (los materiales y productos a emplear) para así paliar los daños y alteraciones que presente, permitiéndonos conservar y recuperar para transmitirla a generaciones futuras. En la Puerta de Córdoba, se llevaron a cabo una serie de estudios que han tenido como objeto la identificación de los materiales constitutivos, características y comportamiento, así como la búsqueda de los productos, técnicas a emplear y materiales -realizados en el Departamento de Análisis del Centro de Intervenciones del I.A.P.H.- y que han marcado la pauta de la actuación posterior.

Los materiales empleados en la construcción de la puerta, fundamentalmente son dos: piedra procedente de los Alcores, calcarenita de color amarilloanaranjado, y ladrillos aplantillados con distinta dimensión y geometría. En su decoración encontramos jarrones constituidos de dos materiales, piedra y material cerámico y lápidas de mármol.

El estado de conservación' que presentaba era malo, (foto I) aunque no existían daños que perjudicaran gravemente su estructura. Las patologías que afectaban a este edificio obedecían a distintos factores, tanto endógenos como exógenos; entre los primeros podemos enumerar:

- Los componentes de la piedra del Alcor (calcarenita de grano grueso y restos fósiles), que la hacen muy porosa, y con poca solidez.

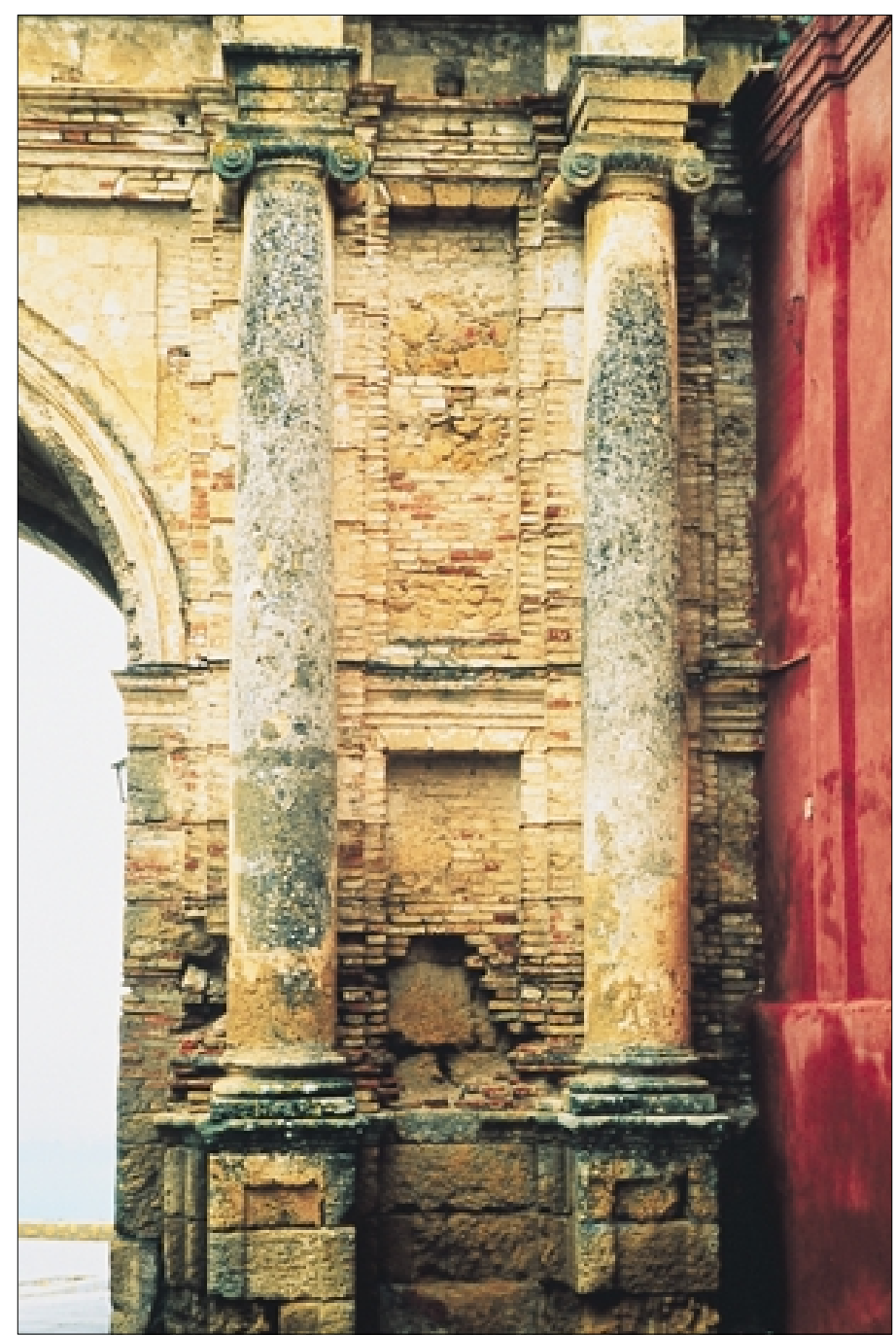

I. Vista de la fachada oeste, lateral derecho, en las que se pueden observar las distintas patologías que afectan a la piedra alcoriza, fábrica de ladrillo y revestimientos. 


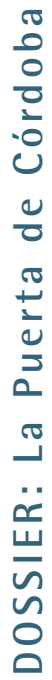

2. Prueba de color en el mortero de rejuntado

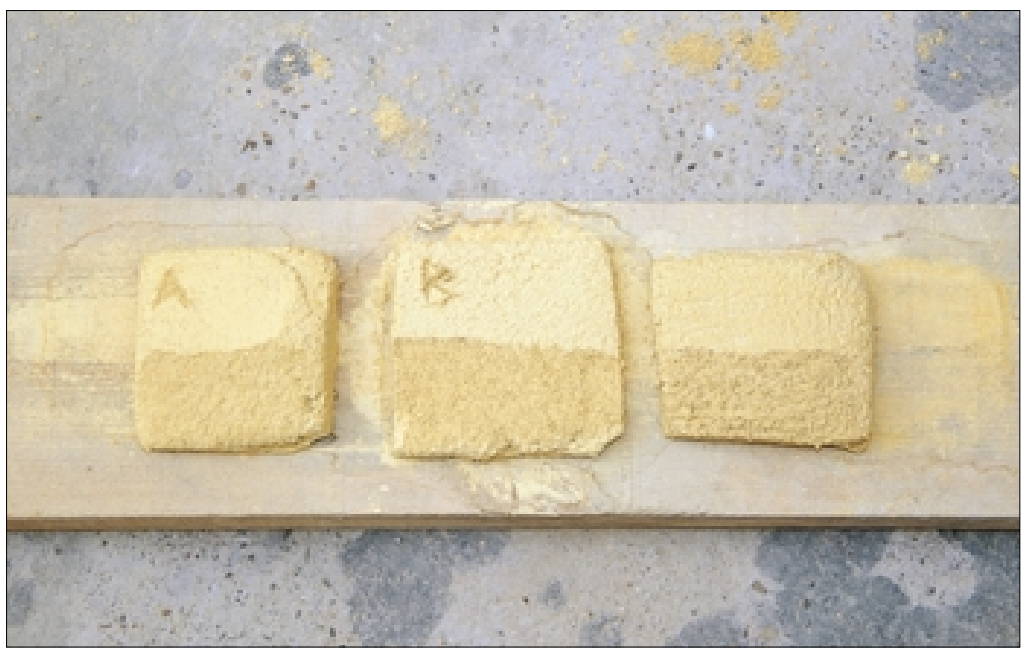

bilidad. Se ha llevado a cabo en zonas que presentaban arenización, disgregación del material y formación de huecos. Se aplicó el consolidante -ester de ácido silícico- de peso molecular muy bajo, que al ser depositado sobre la piedra deteriorada penetra hasta el núcleo sano de ella, reacciona con la humedad del aire y forma gel de ácido silícico. Por estar combinado con siloxanos, el consolidante $\mathrm{OH}$ confiere propiedades hidropelentes, se aplicó con brocha y spray.

Después del tratamiento el material recuperó su solidez y porosidad, y se pudo proceder a la eliminación en seco de zonas que presentaban ataques biológicos, eliminando líquenes y musgos mediante cepillos de cerda y de alambre, para finalmente comenzar el tratamiento de limpieza en húmedo, mediante agua atomizada y cepillado. Durante esta fase se sanearon fisuras y llagas. Una vez suprimida la materia disgregada, los depósitos, el polvo y la suciedad, comenzó el tratamiento de reposición de juntas, realizándose pruebas de color para el mortero de rejuntado de sillares, y de esta forma conseguir una tonalidad similar a la del paramento donde se iba a emplear (foto 2); sólo se aplicó en las que presentaron abofamiento o perdida de material. Se rellenaron con mortero de cemento blanco, cal y marmolina de dosificación 1:2:8 a la que se añadió pigmento natural.

Otro aspecto importante es la recuperación de masa y volumen, lográndose mediante el empleo: $1^{\circ}$ de piedra nueva -con las mismas características de la utilizada originariamente en la puerta- en la reposición de sillares, y $2^{\circ}$ con morteros de recrecidos de dosificación 1:3, adición de resina acrílica (primal AC-33) al $2 \%$ y tierras naturales, utilizando como armadura varillas de acero inoxidable roscado y encofrado en zonas de grandes pérdidas.

Para la integración de las reposiciones se le proporcionó una textura similar a la zona circundante, con textura abujardada. Posteriormente se procedió a la reintegración del color, a un nivel más bajo que la superficie original como criterio diferenciador, y con la intención de hacerlos fácilmente identificable de cerca; en la reintegración con pátinas se empleó pigmentos naturales disueltos en agua, y resina sintética acrílica diluida al 2\%. Finalmente se procedió a la consolidación de la piedra alcoriza, para incrementar la resistencia a la compresión y disminuir la succión capilar, utilizando el producto aplicado anteriormente, basado en ester de sílice (consolidante $\mathrm{OH}$ ), y a la hidrofugación con un producto basado en siloxano modificado (Tegosivín HL - 100), que permite la absorción de agua en estado de vapor.

Gran parte de la obra está realizada con ladrillos aplantillados, existiendo una considerable cantidad de modelos, dimensiones y formas. Fue necesario efectuar un inventario que recogiera todas las características de cada uno de los elementos, para posteriormente encargar el número de cada pieza que se necesitaba en la restauración del edificio. En cuanto al tratamiento efectuado en el 
material cerámico, se comenzó con la realización de catas de limpieza en distintas zonas con diversos tipos de cepillos y espátulas. Este proceso se efectuó de arriba abajo, utilizando espátula, cepillos metálicos (donde el desarrollo biológico era mayor), de cerda y bisturíes (para eliminar depósitos superficiales)

Una vez concluida la limpieza, se comenzó a reponer la fábrica de ladrillo en ambas fachadas, colocando piezas que anteriormente se habían desmontado, y las de nueva manufactura, hasta recuperar los volúmenes perdidos. En la zona de balaustrada, la reintegración empezó colocando las hiladas más bajas hasta llegar al nivel del pretil, rematando el conjunto ladrillos de gotera y solería. La zona de cornisas (foto 5) era la que presentaba mayores daños en elementos decorativos y molduras, por la oxidación de vástagos y refuerzos metálicos que habían provocado pérdidas y caídas de fragmentos de las cornisas. Su reconstrucción se realizó eliminando los elementos metálicos, reemplazándolos por varillas de acero inoxidable y sustituyendo el ladrillo de mesa por piezas ejecutadas in situ mediante un entramado de armadura. Una vez fraguado se procedió a la colocación de las piezas cerámicas en hiladas hasta recuperar la totalidad de las cornisas (foto 6).

Teniendo en cuenta las conclusiones obtenidas en el estudio de correspondencias realizado anteriormente, se inició el revestimiento de la fábrica de ladrillo con un estuco grueso de tonalidad ocre-amarillo, coloreado en masa. Es un revoco de tonalidad similar al de los restos existentes en La Puerta de Córdoba, que han sido respetados e integrados en este proceso.

\section{Elementos singulares}

Decorando la puerta encontramos jarrones y cinco lápidas de piedra. Previo a la intervención en los jarrones se procedió a su catalogación y clasificación según localización, hallándose doce de los veinte que originariamente formaban el conjunto. El mal estado que presentaban los jarrones, con alteraciones, pérdidas y desprendimientos de los elementos que los componían (foto 7), obligó a su levantamiento con medios manuales, eliminando los anclajes de hierro que los sostenían, causantes en gran medida de sus deterioros. Los situados en los pretiles eran los que mostraban mayores daños y pérdidas. La mayoría de las piezas que faltaban en los jarrones correspondían al remate del material cerámico que los coronaban; el resto es material pétreo, que se encontraba fracturado y en estado de arenización.

\section{Piezas que hay que reconstruir y su ubicación}

Orden de colocación: la pieza inferior es identificada
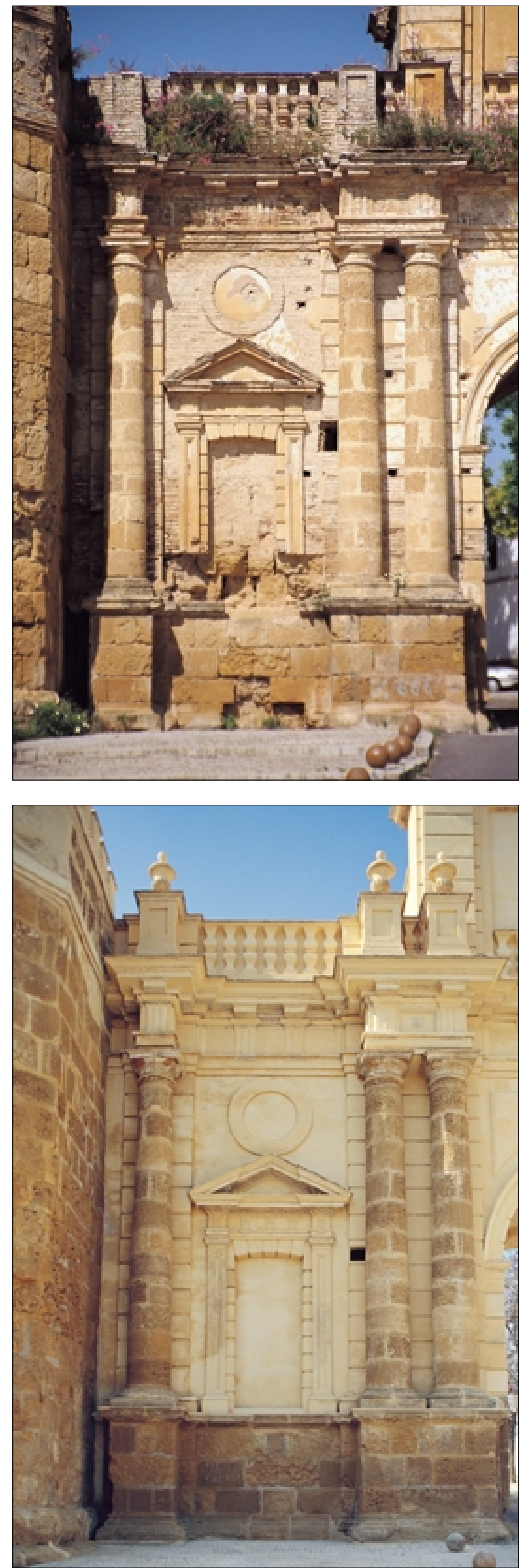

3. Fachada este, lateral izquierdo antes de iniciar la intervención.

4. Vista de la fachada este una vez concluidas las distintas fases de restauración, se pueden observar la reposición de piedra, mortero y reintegración por medio de veladuras. 


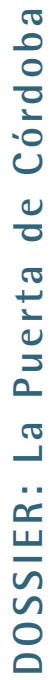

5. Detalle de la cornisa de la fachada oeste antes de la intervención.

6. Detalle de la cornisa de la fachada oeste concluida la restauración.
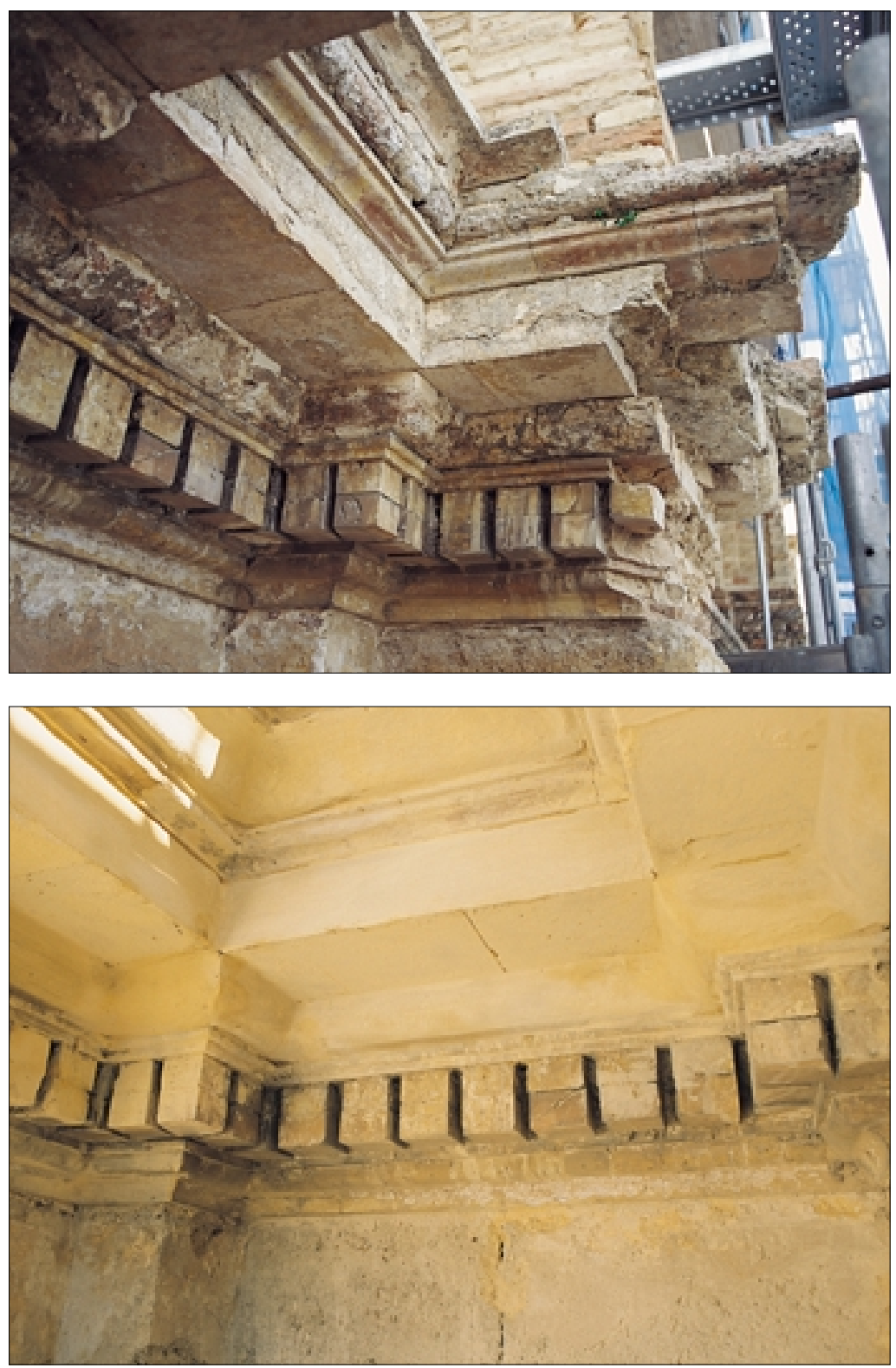

como I y la superior como X. Piezas de material pétreo: I, II y III; piezas de material cerámico: IV, V, VI, VII, VIII, IX y X. La pieza $N^{\circ}$ XI no existía, pero la configuración morfológica del jarrón justifica su presencia. Sólo había que reconstruir las piezas que aparecen numeradas; las casillas en blanco indican piezas completas, por lo que no se recoge su identificación.

Comenzó el proceso de restauración de los jarrones, suprimiendo el material de agarre, posteriormente se cepillaron en seco para eliminar líquenes y se sumergieron las piezas en solución biocida de agua y New Dess al 10\%, durante 24 horas (foto 8).

Se sellaron las piezas rotas mediante resina epoxi en pasta y se recrecieron las pérdidas de soporte con mortero. La reproducción de las piezas que forman los jarrones, se realizó mediante vaciado simple con mortero, repetidas en serie, a partir de un molde obtenido de una pieza original, hasta obtener veintiuna $(2 \mathrm{l}$ ) piezas completas (con la finalidad de ubicarlas donde originariamente se emplazaban, y depositar un jarrón original en el interior del ático, donde posteriormente está previsto su adecuación a espacio museográfico). Del estudio aplicado a su volumen y forma, se dedujo la necesidad de una pieza semiesférica $\left(N^{\circ} \mathrm{XI}\right)$ que rematara y cubriera la superficie del $N^{\circ}$ X y el vástago interior. Concluida la reproducción de los elementos que componían los jarrones, se procedió a su colocación sobre las basas, anclados a la fábrica con varilla roscada de acero inoxidable y sujetos con resina epoxi, se procedió a su reintegración cromática por medio de veladuras de pigmentos obtenidos de tierras naturales (foto 9), posteriormente se consolidó e hidrofugó, para que la cohesión de la pieza perdure.

El proceso de intervención que se realizó sobre el grupo de cinco lápidas situadas en el conjunto -dos en el alzado este, dos en el oeste y una en el vano central-consistió, en eliminar la suciedad superficial de las situadas en el vano central y alzado este, con brochas de pelo corto y bisturí, limpieza de los anclajes metálicos y supresión de óxidos en los bordes de los pernos, (se detectaron fisuras en la lápida situada en el ático del alzado este). Después, en 


\begin{tabular}{|c|c|c|c|c|c|c|c|c|c|c|c|c|c|c|c|c|}
\hline$X I$ & FE4 & FE5 & P02 & F03 & PEI & PE2 & PE3 & PE4 & PE5 & PE6 & P01 & P02 & P03 & P04 & P05 & P06 \\
\hline$x$ & FE4 & FE5 & F02 & F03 & PE1 & PE2 & PE3 & PE4 & PE5 & PE6 & P01 & P02 & P03 & P04 & P05 & P06 \\
\hline IX & FE4 & FE5 & F02 & $\mathrm{F} 03$ & PE1 & PE2 & PE3 & PE4 & PE5 & PE6 & P01 & $\mathrm{P} 02$ & $\mathrm{P} 03$ & P04 & P05 & P06 \\
\hline VIII & FE4 & FE5 & F02 & F03 & PE1 & PE2 & PE3 & PE4 & PE5 & PE6 & P01 & P02 & P03 & P04 & P05 & P06 \\
\hline VII & & FE5 & & & PE1 & PE2 & PE3 & PE4 & PE5 & PE6 & P01 & P02 & P03 & P04 & P05 & P06 \\
\hline VI & & FE5 & & & PE1 & & PE3 & PE4 & PE5 & PE6 & & & & P04 & P05 & P06 \\
\hline V & & FE5 & & & & & & PE4 & PE5 & PE6 & & & & P04 & P05 & P06 \\
\hline IV & & FE5 & & & & & PE3 & PE4 & PE5 & PE6 & & & & P04 & P05 & P06 \\
\hline III & & FE5 & & & & & PE3 & PE4 & PE5 & PE6 & & & & $\mathrm{P} 04$ & P05 & P06 \\
\hline II & & & & & & & & PE4 & PE5 & PE6 & & & & P04 & P05 & P06 \\
\hline I & & & & & & & PE3 & PE4 & PE5 & PE6 & & & & P04 & P05 & P06 \\
\hline
\end{tabular}

las lápidas del tímpano del alzado oeste, se inició el tratamiento biocida y el de limpieza en húmedo debido al fuerte ataque de líquenes que presentaban (foto 10). Finalmente se selló el perímetro de las mismas mediante mortero.

\section{Revestimientos y acabados}

Los revestimientos aplicados a la arquitectura no han tenido gran trascendencia dentro de la investigación histórico-artística, la bibliografía existente es muy escasa, no han sido valorados como elemento material a tener en cuenta por su significación e importancia. Su misión consiste en resguardar y/o adornar una superficie y consecuentemente influyen en la imagen de ese objeto que contemplamos. Desempeñan una función muy importante como protectores, pero no se debe olvidar que son un pilar fundamental para el reconocimiento de esa obra que ha sido intervenida. Sus influencias son decisivas en el aspecto final y no pueden dar lugar a una imagen diferente, sino posibilitar al individuo que la contemple el reconocimiento de ella misma. Es muy importante subrayar la relación de pertenencia de los objetos con las personas que conviven con ellos.

Probablemente, La Puerta de Córdoba presentara una imagen parecida a la que ahora podemos contemplar a partir de su re/construcción en el periodo neoclásico, con la particularidad de que los revestimientos originales de la piedra están prácticamente perdidos. Una vez obtenidos los resultados del estudio de correspondencia de enlucidos, sabemos que la visión global con la tonalidad que hoy presenta es similar a la imagen proyectada antes de su deterioro. Pero esta Puerta que a lo largo de su historia ha cambiado de configuración, también ha podido cambiar de imagen y puede que en algún instante nos mostrara otra cara, influenciada por algo de lo que indudablemente no está exenta ninguna obra de arte: la moda.
7. Estado de conservación de base de jarrón,

pieza $n^{\circ} \mathrm{I}$.

8. Proceso de limpieza, con eliminación de líquenes en pieza cerámica $n^{\circ} \mathrm{V}$.
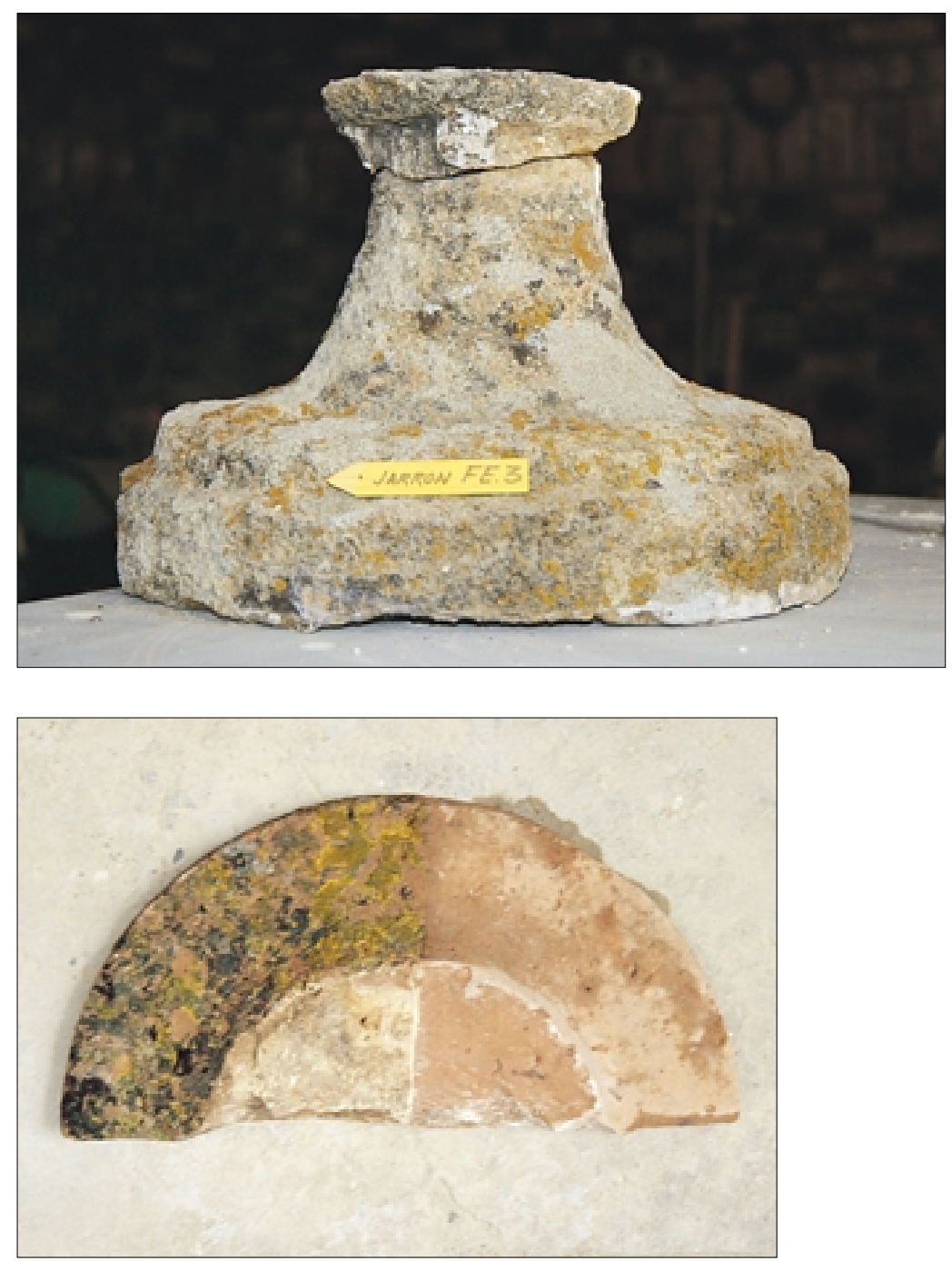


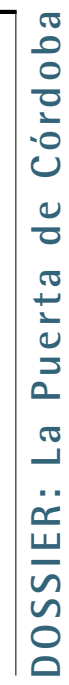

9. Jarrones situados en su emplazamiento original, concluido su proceso de restauración.

10. Detalle de limpieza en lápida situada en el ático de la fachada oeste.

II. Detalle de restos de enlucido situados en la torre sur.
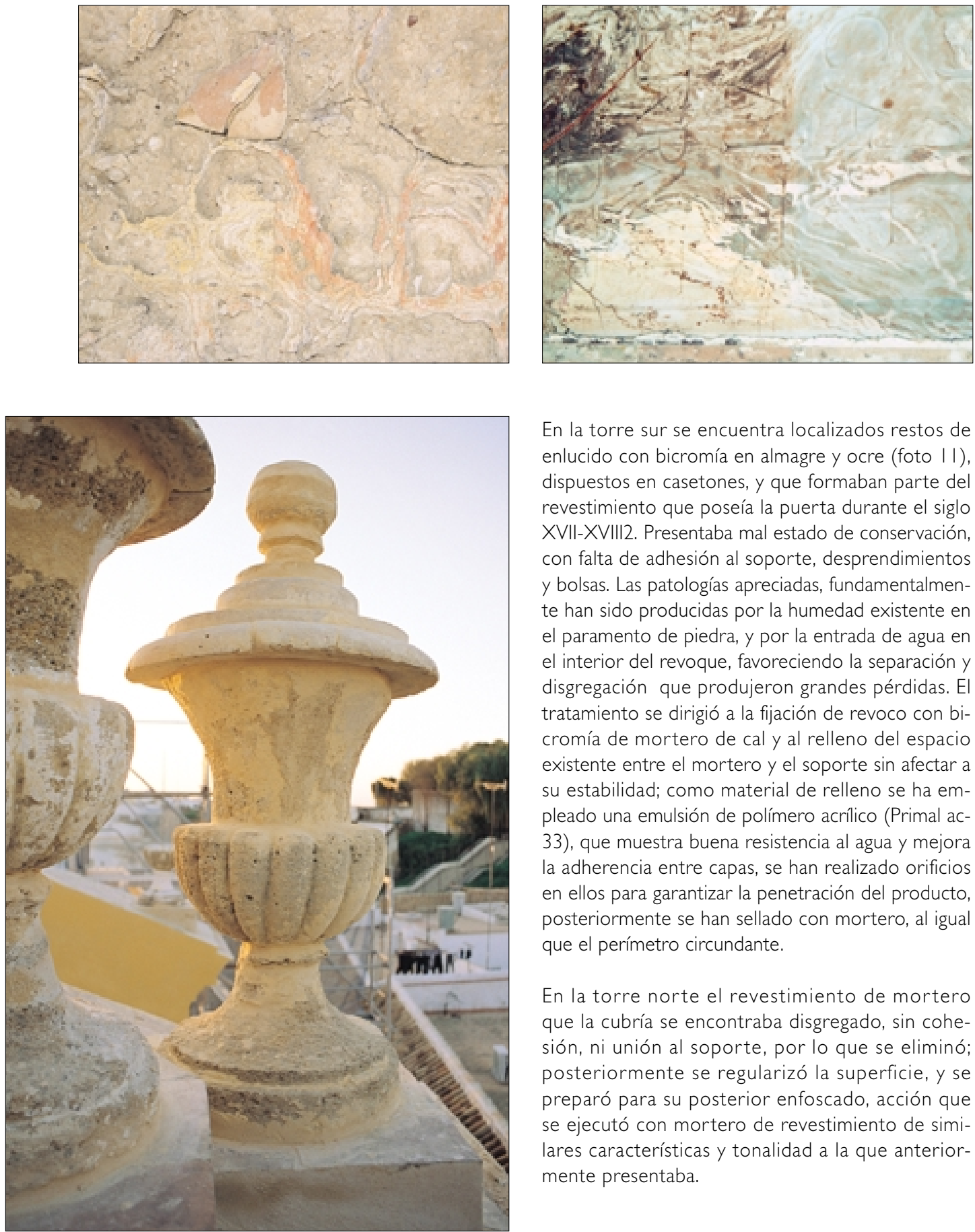

En la torre sur se encuentra localizados restos de enlucido con bicromía en almagre y ocre (foto I I), dispuestos en casetones, y que formaban parte del revestimiento que poseía la puerta durante el siglo XVII-XVIII2. Presentaba mal estado de conservación, con falta de adhesión al soporte, desprendimientos y bolsas. Las patologías apreciadas, fundamentalmente han sido producidas por la humedad existente en el paramento de piedra, y por la entrada de agua en el interior del revoque, favoreciendo la separación y disgregación que produjeron grandes pérdidas. El tratamiento se dirigió a la fijación de revoco con bicromía de mortero de cal y al relleno del espacio existente entre el mortero y el soporte sin afectar a su estabilidad; como material de relleno se ha empleado una emulsión de polímero acrílico (Primal ac33), que muestra buena resistencia al agua y mejora la adherencia entre capas, se han realizado orificios en ellos para garantizar la penetración del producto, posteriormente se han sellado con mortero, al igual que el perímetro circundante.

En la torre norte el revestimiento de mortero que la cubría se encontraba disgregado, sin cohesión, ni unión al soporte, por lo que se eliminó; posteriormente se regularizó la superficie, y se preparó para su posterior enfoscado, acción que se ejecutó con mortero de revestimiento de similares características y tonalidad a la que anteriormente presentaba.

\section{Notas}

I. Sobre el estado de conservación, cfr. TEJEDOR CABRERA, A.: El proyecto de restauración de la Puerta de Córdoba. Metodologías y respuestas. En Boletín del Instituto Andaluz del Patrimonio Histórico, $n^{\circ} .22$, pp 50-66.
2. Sobre el estudio de enlucidos, cfr. OJEDA CALVO, R. Y TABALES RODRÍGUEZ, M. A.: La investigación arqueológica en Bienes Inmuebles. Metodología aplicada en la Puerta de Córdoba. En Boletín del Instituto Andaluz del Patrimonio Histórico, nº. 15, pp 41-52. 